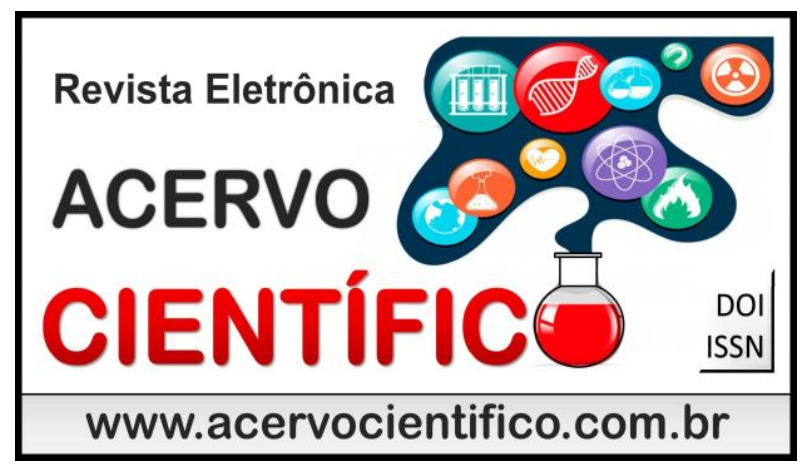

REVISÃo

Recebido em: 4/2019

Aceito em: 5/2019

Publicado em: 8/2019

\title{
Novas tecnologias educacionais aplicadas ao ensino odontológico brasileiro: uma revisão bibliográfica
}

\author{
New educational technologies applied to brazilian dental education: a review \\ Nuevas tecnologías educacionales aplicadas a la educación odontologica brasileña: una \\ revisión bibliográfica
}

Evandro Franco da Rocha ${ }^{1 *}$, Valéria Campanelli Franco da Rocha1.

\begin{abstract}
Resumo: Este trabalho se propôs de forma narrativa a revisar na literatura estudos que envolvem a utilização do ensino à distância em odontologia no Brasil, quais são suas metodologias e as ferramentas desenvolvidas para aplicação na graduação e pós-graduação. A grande maioria dos cursos de atualização "via internet" são em sua grande maioria transcrições do ensino presencial com formatos de alta acessibilidade e baixa interatividade. Existe uma oferta mínima de cursos de atualização e especialização em Odontologia na modalidade à distância. Dos agentes deste novo processo, os alunos de graduação estão capacitados a utilizar ferramentas para educação à distância e obtêm um melhor aproveitamento do curso com elas. O docente, entretanto, ainda necessita de dimensão técnica básica, aprender a utilizar as ferramentas, para desenvolver o curso à distância e do aspecto pedagógico, atuar como facilitador e mediador do processo de ensino-aprendizagem deixando de ser o centro dele.
\end{abstract}

Palavras-chave: Ensino a distância, Ensino semipresencial, Odontologia.

\begin{abstract}
This work proposed in a narrative way to review in the literature studies that involve the use of distance education in dentistry in Brazil, what are its methodologies and the tools developed for application in undergraduate and graduate studies. The vast majority of refresher courses "via the internet" are mostly transcriptions of face-to-face teaching with formats of high accessibility and low interactivity. There is a minimum offer of updating courses and specialization in Dentistry in the distance modality. From the agents of this new process, the undergraduate students are able to use tools for distance education and obtain a better use of the course with them. The teacher, however, still needs a basic technical dimension, to learn how to use the tools, to develop the distance course and the pedagogical aspect, to act as a facilitator and mediator of the teaching-learning process, being no longer the center of it.
\end{abstract}

Keywords: E-Learning, Blended learning, Dentistry.

\footnotetext{
1 Universidade Regional de Blumenau (FURB), Blumenau- SC. *E-mail: efrocha@furb.br
} 
Resumen: Este trabajo se propuso de forma narrativa a revisar en la literatura estudios que involucran la utilización de la enseñanza a distancia en odontología en Brasil, cuáles son sus metodologías y las herramientas desarrolladas para aplicación en la graduación y posgrado. La gran mayoría de los cursos de actualización "vía internet" son en su gran mayoría transcripciones de la enseñanza presencial con formatos de alta accesibilidad y baja interactividad. Hay una oferta mínima de cursos de actualización y especialización en Odontología en la modalidad a distancia. De los agentes de este nuevo proceso, los alumnos de graduación están capacitados para utilizar herramientas para educación a distancia y obtienen un mejor aprovechamiento del curso con ellas. El docente, sin embargo todavía necesita de dimensión técnica básica, aprender a utilizar las herramientas, para desarrollar el curso a distancia y del aspecto pedagógico, actuar como facilitador y mediador del proceso de enseñanza-aprendizaje dejando de ser su centro.

Palabras clave: Educación a distancia, Educación semipresencial, Odontología.

\section{INTRODUÇÃO}

A sociedade contemporânea tem imposto desafios constantes aos setores da saúde e da educação, traduzidos na exigência de revisões e reformulações na política de formação de recursos humanos. $O$ volume do conhecimento se multiplicou e o resultado é uma compressão absurda de informações nos cursos de graduação e também pós-graduação em Odontologia, com sobrecarga do cognitivo e pulverização do conhecimento (DIAS HS et al., 2013).

A formação do odontólogo no Brasil deveria dar a este a capacidade de identificar problemas relevantes à sua volta, avaliar posições quanto a esses problemas e atuar junto à sociedade, modificando a realidade atual. Nessa perspectiva, além de preparar para uma atuação profissional plena, os cursos de odontologia deveriam preparar para o enfrentamento das dificuldades colocadas pela experiência da vida em sociedade, trabalhando competências gerais que permitam aos estudantes a atualização contínua, e não apenas a aquisição imediata de informações que, com o tempo, tornam-se obsoletas (SETTE SPH et al., 2015).

Não é isto que ocorre na realidade, em parte esta situação pode ser justificada pela cultura arraigada do ensino centrado no professor, como mero repassador de conteúdos ao invés de atuar como facilitador e mediador do processo de ensino-aprendizagem. Para vencer este problema sugere-se a utilização de estratégias educacionais inovadoras, caracterizadas pela temática em torno de problemas, pela integração de conteúdos e ênfase no desenvolvimento cognitivo e das habilidades, além do aprendizado contextual. (LIMA VV et al., 2015).

Com esta finalidade, diversos novos recursos ou tecnologias educacionais, disponíveis por meio da Internet, podem ser utilizados com vistas a alcançar maior clareza e precisão na aquisição do conhecimento, além de permitir o envolvimento do aluno de forma mais efetiva ao curso e ao tema. $\mathrm{O}$ aluno deve ser capacitado a entender e utilizar inovações tecnológicas, com visão crítica e fundamentada cientificamente, em contrapartida a modelos de formação mecanicista e tecnicista (REZENDE F, 2000).

Também em virtude do rápido desenvolvimento do conhecimento, o odontólogo necessita aprendizagem e aperfeiçoamento constantes e as novas tecnologias educacionais como o ensino à distância, são alternativas que podem ser utilizadas para suprir esta necessidade. Considerando ainda fatores como falta de tempo, alto custo e dificuldade ou falta de disponibilidade de locomoção, inerentes à profissão, o ensino à distância poderia oferecer uma alternativa viável para a educação ou formação continuada dos profissionais em saúde no geral. No entanto, a odontologia no Brasil, ainda tem poucos cursos utilizando novas tecnologias educacionais, como o ensino a distância, via web, embora algumas ferramentas tecnológicas ou de informática, facilitadoras à atuação do professor, sejam utilizadas (D’ALPINO PHP. et al., 2018).

Novas tecnologias educacionais com o apoio do ensino a distância e web podem potencializar 0 processo de ensino e aprendizagem, uma vez que essas alternativas pedagógicas compartilham 
características comuns, tais como, centrar a aprendizagem no aluno, desenvolver habilidade de comunicação e aquisição do conhecimento de forma autônoma, além de permitir o acesso à informação, o acompanhamento das turmas, a interatividade entre os alunos e entre os alunos e o professor, facilitando a colaboração e, consequentemente, o envolvimento dos alunos com as tarefas de aprendizagem, contribuindo para o aprimoramento do aprendizado (SILVA ICS et al., 2017).

É necessária, uma visão atualizada sobre estas novas tecnologias educacionais e as formas nas quais elas estão sendo aplicadas, suas finalidades e resultados obtidos no ensino da odontologia no Brasil. Estarão os alunos e os professores de odontologia capacitados a utilizá-las? Qual o estado atual desta arte no Brasil?

\section{REVISÃo}

A educação formal, que já flertava com metodologias diferenciadas do ensino tradicional de transmissão de conteúdo, diante de novas ferramentas e tecnologias de comunicação, como o telefone celular, aplicativos para comunicação de texto ou voz, imagem em tempo real, plataformas virtuais para redes sociais, surgidas na última década, agora se vê na necessidade de incorporação destas novas tecnologias no processo de aprendizagem. Uma destas incorporações dá-se através do desenvolvimento da educação à distância. Um grande número de indivíduos migrou para esta modalidade de ensino, tanto que as Universidades, cientes desta realidade, investem na expansão e oferta de cursos de graduação e pósgraduação à distância em todo o Brasil (ALVES L, 2011).

$\mathrm{Na}$ área do ensino odontológico, Jardim JJ et al. (2001) afirmaram ser crescente a oferta e a procura por educação continuada ou permanente através de ensino à distância (EAD) utilizando-se como recurso as redes informatizadas. Entretanto, Masoti AF et al. (2001), embora concordem que estes cursos de educação continuada em odontologia venham se tornado cada vez mais importantes para a disseminação de informações e habilidades, argumenta que os números sobre a educação continuada em Odontologia no Brasil não estão disponíveis.

Masoti AS et al. (2002) estudaram a EAD aplicada aos cursos de educação continuada em odontologia, através da busca de sites dedicados a EAD em odontologia na língua portuguesa. Foram encontrados 10 cursos em odontologia oferecidos por EAD no Brasil, o que representa menos de $10 \%$ da oferta de cursos similares nos Estados Unidos da América.

Garbin D et al. (2010) pesquisaram no ano de 2006 o que a Internet oferecia aos cirurgiões-dentistas no que se referia aos tipos ou modalidades de curso de educação continuada em odontologia. Os autores selecionaram oito sites que ofereciam educação continuada em odontologia via web. Dentre os cursos oferecidos, apenas um tinha nível de especialização, na área de odontologia em saúde coletiva. Os restantes eram cursos de aperfeiçoamento, atualização ou extensão.

Com relação à utilização do ensino a distância como coadjuvante do ensino presencial nos cursos de graduação em Odontologia, algumas experiências foram realizadas com resultados bem variáveis, dependendo do tipo de ferramenta e interação utilizadas (D'ALPINO PHP. et al., 2018).

Corrêa L (2001) investigou os alunos de vários cursos de odontologia do Brasil, submetidos a sites de ensino de patologia geral e bucal, utilizados como apoio ao ensino presencial. Segundo a autora, os alunos estão aptos a utilizar a internet como ferramenta de ensino na modalidade site ou hipertexto e que aprovaram a iniciativa. Entretanto, as instituições devem estar bem preparadas do ponto de vista de infraestrutura, incluindo-se aí o preparo de recursos humanos e estratégias pedagógicas.

Arriada MC et al. (2005), descreveram a experiência da Universidade de Santa Cruz do Sul em busca de um processo mais inclusivo de formação de professores para Educação a Distância. Nestes processos, buscaram que todos os indivíduos envolvidos participassem de forma ativa da EAD. Isto permitiu que fossem identificadas as formas mais importantes que deveriam participar da formação dos professores, para que estes não subutilizassem o potencial do EAD. 
Skelton MMC et al. (2007), avaliaram o desempenho de alunos da disciplina de Endodontia do curso de Graduação em Odontologia da Faculdade São Leopoldo Mandic, Campinas - São Paulo, que contaram com uma plataforma educacional para apoio ao ensino presencial. O material de apoio disponibilizado na plataforma contou com apresentações, apostilas, hipertexto, perguntas frequentes, diretrizes para 0 desenvolvimento de trabalhos, avaliações e tutoriais animados, projetados de maneira interativa. Os alunos apresentaram uma média de acesso $128,75 \%$ superior ao esperado e com respeito ao desempenho alcançado pelos alunos, observou-se que os que mais acessos fizeram ao material didático disponibilizado obtiveram melhor desempenho geral, avaliados através das médias semestrais na disciplina.

Hoshikawa MH et al. (2009) utilizaram a plataforma MOODLE como apoio ao ensino presencial em diversas disciplinas de um curso de odontologia. Através do registro das participações dos alunos nas atividades interativas puderam avaliar o grau de participação dos mesmos nas atividades das disciplinas. Os resultados obtidos levaram-no a concluir que o aluno tem dificuldade em compreender a finalidade do ensino a distância na graduação em odontologia, e apresentaram baixa interatividade no ambiente virtual.

Um estudo com a intenção de analisar a viabilidade de utilização da Internet como apoio ao ensino presencial foi dirigido por Nogi FM e Melani RFH (2009). Os autores realizaram uma avaliação de alunos do curso de Odontologia da Faculdade de Odontologia da Universidade de São Paulo através de questionário, visando obter informações quanto ao preparo dos alunos no manejo do computador e da Internet. Os resultados levaram os autores a concluir que os alunos apresentavam boa aptidão no manejo dos computadores e da Internet, podendo utilizá-la como ferramenta de auxílio pedagógico em sua formação.

Franco LLMM et al. (2009), cita que a maior dificuldade é saber utilizar as ferramentas que compões o $E A D$, principalmente para docentes com mais tempo de carreira. Ainda segundo estes autores o processo de facilitador do ensino deve ser exercido por estes docentes com viés ao EAD. Logicamente isto envolve 0 professor se reinventar em termos de linguagem e até mesmo valores, que deverão ser aprendidos dentro deste novo arsenal tecnológico e aplicados de maneira compatível com os estudantes que frequentam as Instituições de Ensino Superior (idem).

Espejo TLC et al. (2010) utilizaram uma ferramenta via web, com o intuito de apoiar o ensino presencial da Disciplina de Dentística Operatória da Faculdade de Odontologia da Universidade de São Paulo. Aos alunos foi aplicado um questionário para avaliação da iniciativa, que foi considerada efetiva por $98,2 \%$ dos mesmos. A comparação entre as médias finais das turmas participantes e não participantes do projeto mostraram diferença estatisticamente significante. Segundo os autores os alunos têm interesse por essa ferramenta de ensino, porém a mudança na abordagem deve ser um processo gradual, mas que deve ser aplicado como forma complementar ao ensino presencial.

Para utilização do ensino à distância como forma de educação continuada, Camargo LB (2010) desenvolveu um curso de extensão para capacitação profissional de cirurgiões-dentistas da rede pública na execução do Tratamento Restaurador Atraumático (ART). Segundo os autores, se desenvolvidos adequadamente, os cursos de capacitação à distância apresentam potencial para promover as competências necessárias à correta execução de técnicas odontológicas, como por exemplo, o ART (Camargo LB, 2010).

Costa FOC et al. (2010) analisaram a informática como ferramenta de ensino em Odontologia, destacando dentre as possibilidades de utilização da EAD em odontologia: a apresentação antecipada do conteúdo da aula, utilização do IRC (Internet Relay Chat, Bate-papo pela Internet) para através de horário e canal específico os alunos poderem debater o conteúdo exposto, vídeo conferência com participação de professores convidados, newsgroups (listas de discussão), que permitem a troca de experiências com professores e colegas e ainda salas de aula virtuais.

Cunha AIMZ et al. (2012) estudaram alunos do curso de Odontologia (UFPB) que cursaram a disciplina oferecida presencialmente, porém com suporte da ferramenta Moodle. Os resultados mostraram que embora a grande maioria dos alunos refletisse que a utilização do ambiente virtual de aprendizagem fosse importante e tenham tido melhora em seu desempenho, a interatividade entre eles apresentou-se deficiente, 
pois apenas $21 \%$ se dispuseram a explicar suas ideias aos demais colegas. Concluíram que houve deficiente na inteiração dos alunos e a necessidade de propostas para sobrepujar isto.

Dotta EAV et al (2012), propuseram uma metodologia para montagem de um curso de 14 módulos de odontologia utilizando a plataforma Moodle. A elaboração foi dividida em planejamento pedagógico e inserção do conteúdo dentro da plataforma. Embora o Moodle tenha permitido a elaboração do curso de forma satisfatória, os autores sugerem que os envolvidos necessitam domínio dos conceitos básicos de informática e das ferramentas da plataforma.

Silva RHAS, et al. (2015) relatam que a Universidade de São Paulo, Campus de Ribeirão Preto, utiliza uma ferramenta chamada de Teleodontologia. Segundo os autores a Teleodontologia é uma ferramenta virtual, que objetiva facilitar o ensino e aprendizado da graduação. Na prática funciona como um portal, onde todo o conteúdo é disponibilizado pelos docentes e ao qual os alunos acessam, uma vez matriculados na disciplina, por meio de login e senha, não diferindo muito de outras experiências em portais de Ambiente Virtual de Aprendizagem

D'alpino PHP et al. (2018) fornecem em seu artigo generalidades de diversas ferramentas disponíveis para o aprendizado apontando diretrizes para estudos futuros relacionados aos métodos de ensino e aprendizagem em profissões da área de saúde, principalmente Odontologia e ressaltam que a maior dificuldade á a crítica, pela falta de contato pessoal que recai sobre o EAD e busca de soluções para melhorar este vínculo.

Em uma revisão sobre o EAD em odontologia no Brasil, Faleiro FRG e Salvago BM (2018), concluíram que $o$ recurso pode ser utilizado de maneira complementar, apoiando as aulas presenciais e que 0 paradigma do uso da EAD em cursos tradicionais como este vem sendo aos poucos alterado, contemplando novos modos de ensinar e aprender.

\section{CONSIDERAÇÕES FINAIS}

Evidencia-se através do exposto, que a oferta de cursos de odontologia bem estruturados, na modalidade EAD, no Brasil, é bastante restrita quando se fala de capacitação profissional através de educação continuada ou especialização. Muitos cursos chamados de atualização são oferecidos "via internet", mas são em sua grande maioria transcrições do ensino presencial, com a apresentação de "slides" do tema ou a explanação do professor gravada em vídeo, ou seja, com formatos de alta acessibilidade e baixa interatividade. Poderíamos talvez atribuir esta baixa oferta de cursos completos e interativos a: própria falta de formação ou capacitação profissional dos agentes envolvidos desde o planejamento até o suporte ao aluno receio por parte do corpo docente no aplicar das novas tecnologias ou até mesmo à falta de infraestrutura das Instituições para EAD, no que diz respeito à Odontologia.

\section{REFERÊNCIAS}

1. ALVES L Educação a distância: conceitos e história no Brasil e no mundo. Revista Brasileira de aprendizagem aberta e à distância, 2011; 10: 83-92.

2. ARRIADA MC, et al. Aprendendo e ensinando EAD: a importância da vivência na qualificação da formação. Colabor@ - Revista Digital da CVA - Ricesu, 2005; 3(10).

3. CAMARGO LB Estruturação e avaliação de curso de extensão em Tratamento Restaurador Atraumático (ART) utilizando a educação à distância. Tese (Doutorado em Odontopediatria). Faculdade de Odontologia, Universidade de São Paulo, 2010,101p.

4. CORRÊA L Análise da mudança de paradigma do ensino de graduação em odontologia: proposta de ensinoaprendizado à distância via internet. Tese (Doutorado em Patologia). Faculdade de Odontologia, Universidade de São Paulo, 2001, 205p.

5. COSTA FOC, et al. A informática como ferramenta para o ensino da odontologia In: XII CONGRESSO BRASILEIRO DE INFORMÁTICA EM SAÚDE, 2010, Porto de Galinhas. Anais eletrônicos. Porto de Galinhas: SOCIEDADE BRASILEIRA DE INFORMÁTICA EM SAÚDE. 
6. CUNHA-ARAÚJO IMZ et al. Avaliação da percepção dos alunos da disciplina de endodontia sobre o uso do Ambiente Virtual de Aprendizagem (Moodle). Uso do questionário de auto avaliação COLLES. Revista da ABENO, 2012; 12(2):163-169.

7. D'ALPINO PHP, et al. Uso de Plataformas Integradoras de Ferramentas Tecnológicas e Pedagógicas em Ambiente Virtual de Aprendizagem em Profissões de Saúde Revista de Ensino Educação e Ciências Humanas, 2018; 19(2):168-176.

8. DA SILVA, ICS et al. As Novas Tecnologias e aprendizagem: desafios enfrentados pelo professor na sala de aula. Revista Digital em Debate, 2017; 15:107-123.

9. DIAS, HS et al. A trajetória da política nacional de reorientação da formação profissional em saúde no SUS. Ciência e saúde coletiva, 2013; 18(6):1613-1624.

10. DOTTA EAV et al. Elaboração de um curso interativo voltado ao aprendizado de um sistema aplicativo em Odontologia, utilizando a plataforma Moodle. Revista de Odontologia da Universidade Cidade de São Paulo, $2012 ; 24(1): 6-14$.

11. ESPEJO-TRUNG, LC et al. Desenvolvimento de um sistema complementar de educação à distância em Dentística Operatória. Revista brasileira de aprendizagem aberta e a distância, 2010; 9:1-12.

12. FRANCO LLMM, et al. O professor do curso de odontologia: sua formação e os desafios frente às exigências atuais. Revista Profissão Docente.2009; 9(20):57-74.

13. GARBIN D, et al. Educação Continuada em Odontologia Via Web: um Estado da Arte no Brasil. In: CONGRESSO BRASILEIRO DE INFORMATICA EM SAUDE, 2006, Porto de Galinhas. Anais eletrônicos.

14. FALEIRO FRG, SALVAGO BM Educação a distância nos cursos de graduação em odontologia no brasil. Revista Brasileira de Aprendizagem Aberta e a Distância, 2018; 17(1):e45.

15. HOSHIKAWA MH, et al. Ensino a Distância no curso de Odontologia - Relato de experiências. ConScientiae Saúde, 2009; 8(2):301-307.

16. JARDIM, JJ, et al. Educação à distância e odontologia. Parte I: Noções de educação à distância e sua aplicação na odontologia. Jornal de Assessoria ao Odontologista. 2001; 27:45-50.

17. LIMA VV, et al. Ativadores de processos de mudança: uma proposta orientada à transformação das práticas educacionais e da formação de profissionais de saúde. Ciência e saúde coletiva, 2015; 20(1):279-288.

18. MASOTI AS, et al. Ensino a distância em odontologia via internet: o que está sendo produzido no Brasil? Revista Odonto Ciência, 2002; 17(35):96-102.

19. NOGI FM, MELANI RFH Internet como ferramenta de apoio no ensino odontológico: avaliação da aptidão técnica dos estudantes de graduação da Faculdade de Odontologia da Universidade de São Paulo. Saúde, Ética \& Justiça, 2009; 14(2):84-91, 2009.

20. REZENDE F As novas tecnologias na prática pedagógica sob a perspectiva construtivista. Ens. Pesquisa em Educação em Ciências, 2000; 2(1):70-87.

21. SETTE-DE-SOUZA PH, et al. Competências gerais e habilidades específicas: a realidade do ciclo básico. Revista da ABENO, 2015; 15(1):38-47.

22. SILVA RHAS, et al. O uso da Teleodontologia no ensino de Odontologia Legal: relato de experiência Revista da ABENO, 2015; 15(2):95-104, 2015.

23. SKELTON-MACEDO MC, et al. Endodontia na graduação com Endodontia na graduação com ensino presencial e suporte à distância: estratégia motivacional ao estudo individual Revista da ABENO, 2007; 7(1):68-75. 